\title{
Generation of $\mathrm{mAb}$ variants with less attractive
}

\section{self-interaction but preserved target binding by}

\section{well-directed mutation}

Martin Domnowski ${ }^{1,2}$, Ken Lo Presti ${ }^{1}$, Jonas Binder ${ }^{1}$, Josef Reindl ${ }^{2}$, Lucille Lehmann ${ }^{2}$, Felix

Kummer ${ }^{2}$, Meike Wolber ${ }^{2}$, Marion Satzger ${ }^{2}$, Marco Dehling², Jan Jaehrling ${ }^{2}$, Wolfgang Frie $\beta^{1}$

${ }^{1}$ Department of Pharmacy; Pharmaceutical Technology and Biopharmaceutics; Ludwig Maximilians-Universitaet; Munich, Germany

${ }^{2}$ MorphoSys AG; Department of Protein Sciences (Research); Planegg; Germany 


\section{Supporting Information}

Table S1. Peptide list of paratope mapping

\begin{tabular}{|c|c|c|c|c|c|c|}
\hline Chain & start & end & Sequence & time & $\Delta \mathrm{HX}$ & $\mathrm{p}$-value \\
\hline \multirow[t]{30}{*}{ Heavy Chain } & 46 & 58 & EWMGGIIPIYGTA & 1 & -1.08 & 0.001 \\
\hline & 46 & 58 & EWMGGIIPIYGTA & 10 & -0.90 & 0.000 \\
\hline & 46 & 58 & EWMGGIIPIYGTA & 60 & -0.75 & 0.000 \\
\hline & 46 & 58 & EWMGGIIPIYGTA & 240 & -0.66 & 0.000 \\
\hline & 48 & 59 & MGGIIPIYGTAY & 1 & -1.08 & 0.001 \\
\hline & 48 & 59 & MGGIIPIYGTAY & 10 & -0.77 & 0.003 \\
\hline & 48 & 59 & MGGIIPIYGTAY & 60 & -0.81 & 0.000 \\
\hline & 48 & 59 & MGGIIPIYGTAY & 240 & -0.92 & 0.001 \\
\hline & 50 & 59 & GIIPIYGTAY & 1 & -1.09 & 0.001 \\
\hline & 50 & 59 & GIIPIYGTAY & 60 & -0.52 & 0.000 \\
\hline & 50 & 59 & GIIPIYGTAY & 240 & -0.53 & 0.001 \\
\hline & 103 & 111 & YVTYRRYWF & 1 & -0.85 & 0.001 \\
\hline & 103 & 111 & YVTYRRYWF & 10 & -0.80 & 0.009 \\
\hline & 103 & 111 & YVTYRRYWF & 60 & -1.18 & 0.000 \\
\hline & 103 & 111 & YVTYRRYWF & 240 & -1.33 & 0.000 \\
\hline & 103 & 112 & YVTYRRYWFD & 1 & -0.81 & 0.001 \\
\hline & 103 & 112 & YVTYRRYWFD & 10 & -0.79 & 0.009 \\
\hline & 103 & 112 & YVTYRRYWFD & 60 & -1.18 & 0.000 \\
\hline & 103 & 112 & YVTYRRYWFD & 240 & -1.43 & 0.000 \\
\hline & 103 & 113 & YVTYRRYWFDY & 1 & -1.11 & 0.000 \\
\hline & 103 & 113 & YVTYRRYWFDY & 10 & -0.68 & 0.000 \\
\hline & 103 & 113 & YVTYRRYWFDY & 60 & -1.59 & 0.000 \\
\hline & 103 & 113 & YVTYRRYWFDY & 240 & -1.70 & 0.000 \\
\hline & 104 & 113 & VTYRRYWFDY & 1 & -0.80 & 0.000 \\
\hline & 104 & 113 & VTYRRYWFDY & 10 & -0.71 & 0.010 \\
\hline & 104 & 113 & VTYRRYWFDY & 60 & -1.35 & 0.000 \\
\hline & 104 & 113 & VTYRRYWFDY & 240 & -1.64 & 0.000 \\
\hline & 106 & 113 & YRRYWFDY & 1 & -0.45 & 0.000 \\
\hline & 106 & 113 & YRRYWFDY & 60 & -1.09 & 0.000 \\
\hline & 106 & 113 & YRRYWFDY & 240 & -1.35 & 0.000 \\
\hline
\end{tabular}

Criteria for significant differences of $\Delta \mathrm{HX}$ :

Pooled standard deviation $\mathrm{sp}=0.135$, standard error of the mean SEM $=0.11$, Confidence criteria for $\triangle \mathrm{HX}$ values were calculated by following equation: $|\Delta H X|>k \times S E M$ using $\mathrm{k}=-3.75$ according to

Student's t-distribution (One-tailed, $\alpha=0.01, \mathrm{df}=4$ ) 
Table S2. Characterization of $\mathrm{mAb}$ variants generated based on in silico surface analysis Absolute values

\begin{tabular}{|c|c|c|c|c|c|c|}
\hline \multirow[t]{2}{*}{ mAb variant } & \multirow[t]{2}{*}{ Region } & \multicolumn{2}{|c|}{$\begin{array}{c}\text { Self-interaction } \\
{[\%]}\end{array}$} & \multirow{2}{*}{$\begin{array}{c}\text { Off-target } \\
\text { binding } \\
{[\%]}\end{array}$} & \multicolumn{2}{|c|}{$\begin{array}{l}\text { Target binding } \\
\mathbf{K}_{\mathrm{D}}^{\text {app }}[\mathrm{nM}]\end{array}$} \\
\hline & & Average & SD & & Average & SD \\
\hline Wildtype & --- & 100.0 & 13.9 & 100 & 7.7 & 1.0 \\
\hline I52R & \multirow{12}{*}{ HCDR2 } & 83.0 & 4.0 & & 5.9 & 0.8 \\
\hline I53R & & 70.6 & 2.0 & & 6.6 & 0.2 \\
\hline Y54R & & 67.6 & 2.0 & & 7.4 & 0.4 \\
\hline Y58R & & 67.9 & 3.0 & & 7.3 & 0.4 \\
\hline G55R & & 50.0 & 2.0 & 180 & 6.0 & 0.2 \\
\hline G55D & & 66.0 & 8.0 & & 6.8 & 0.6 \\
\hline T56R & & 75.4 & 2.0 & & 5.7 & 0.4 \\
\hline T56D & & 50.4 & 2.0 & 25 & 5.9 & 0.6 \\
\hline $\begin{array}{c}\text { I52A I53A } \\
\text { Y54A Y58A }\end{array}$ & & 127.8 & 3.0 & 8 & 9.7 & 0.5 \\
\hline $\begin{array}{l}\text { I52A I53A } \\
\text { Y54S Y58S }\end{array}$ & & 110.0 & 3.0 & 16 & 11.1 & 0.4 \\
\hline $\begin{array}{c}\text { I52V I53V } \\
\text { Y54A Y58A }\end{array}$ & & 86.2 & 3.0 & 18 & 9.8 & 0.6 \\
\hline $\begin{array}{l}\text { I52V I53V } \\
\text { Y54S Y58S } \\
\end{array}$ & & 63.4 & 3.0 & 12 & 9.7 & 0.6 \\
\hline W100cV & \multirow{2}{*}{ HCDR3 } & 135.5 & 2.0 & 67 & 5.8 & 0.2 \\
\hline W100cS & & 124.8 & 1.0 & 67 & 7.7 & 0.4 \\
\hline G27R & HCDR1 & 48.8 & 2.0 & 161 & 5.4 & 0.7 \\
\hline G27R T56D & HCDR1/HCDR2 & 85.7 & 7.4 & 61 & 6.3 & 0.8 \\
\hline
\end{tabular}


Table S3. Characterization of mAb variants engineered based on HDX-MS analysis Absolute values

\begin{tabular}{|c|c|c|c|c|c|c|c|}
\hline \multirow{2}{*}{$\begin{array}{l}\text { Hot } \\
\text { spot }\end{array}$} & \multirow[t]{2}{*}{ mAb variant } & \multirow{2}{*}{$\begin{array}{c}\text { Domain } \\
/ / \\
\text { Chain }\end{array}$} & \multicolumn{2}{|c|}{$\begin{array}{c}\text { Self-interaction } \\
{[\%]}\end{array}$} & \multirow{2}{*}{$\begin{array}{c}\text { Off-target } \\
\text { binding } \\
{[\%]}\end{array}$} & \multicolumn{2}{|c|}{$\begin{array}{c}\text { Target binding } \\
{[\mathrm{nM}]}\end{array}$} \\
\hline & & & Average & SD & & Average & SD \\
\hline--- & Wildtype & --- & 100.0 & 13.9 & 100 & 7.7 & 1.0 \\
\hline \multirow{11}{*}{1} & Y102D & \multirow{11}{*}{$\mathrm{Fab} / \mathrm{HC}$} & 100.0 & 7.7 & 64 & 5.3 & 0.5 \\
\hline & Q105D & & 96.0 & 8.0 & 120 & 5.3 & 0.7 \\
\hline & L108D & & 88.0 & 5.0 & 118 & 5.3 & 0.5 \\
\hline & Q105D L108D & & 58.6 & 2.3 & 99 & 6.0 & 0.5 \\
\hline & Y102D Q105D L108D & & 81.4 & 1.9 & 38 & 5.6 & 0.6 \\
\hline & Y102R & & 73.2 & 2.0 & 180 & 5.8 & 0.8 \\
\hline & Q105R & & 65.7 & 2.5 & 119 & 7.0 & 0.9 \\
\hline & L108R & & 69.2 & 3.7 & 171 & 6.6 & 0.5 \\
\hline & R100aD & & 76.4 & 1.2 & 1 & 9.6 & 0.6 \\
\hline & R100aD Y102D & & 90.7 & 4.5 & 1 & 11.1 & 0.4 \\
\hline & R100aD R100 & & 77.8 & 2.3 & 1 & 22.1 & 1.0 \\
\hline \multirow{4}{*}{2} & V379D & \multirow{4}{*}{$\mathrm{Fc} / \mathrm{HC}$} & 53.5 & 0.8 & 140 & 4.5 & 0.9 \\
\hline & E380R & & 96.1 & 5.0 & 176 & 6.6 & 0.7 \\
\hline & E382R & & 90.9 & 4.4 & 183 & 7.2 & 0.8 \\
\hline & N384D & & 85.1 & 0.1 & 144 & 7.2 & 0.7 \\
\hline \multirow{8}{*}{3} & S155D & \multirow{8}{*}{$\mathrm{Fab} / \mathrm{LC}$} & 95.2 & 8.8 & 144 & 4.0 & 0.7 \\
\hline & S155R & & 71.6 & 2.8 & 135 & 5.5 & 0.9 \\
\hline & N157D & & 82.4 & 1.5 & 160 & 4.7 & 0.8 \\
\hline & Q159D & & 85.3 & 3.7 & 139 & 4.7 & 0.6 \\
\hline & Q159E & & 95.8 & 1.5 & 140 & 4.7 & 0.6 \\
\hline & T163D & & 90.9 & 4.7 & 144 & 5.1 & 0.7 \\
\hline & T163R & & 93.0 & 3.9 & 151 & 4.7 & 0.6 \\
\hline & S155D N157D Q159D T163D & & 74.1 & 1.8 & 119 & 5.4 & 0.8 \\
\hline
\end{tabular}


Table S4. Peptide list of self-interaction sites mapping

\begin{tabular}{|c|c|c|c|c|c|c|}
\hline Chain & start & end & Sequence & time & $\Delta H X$ & $p$-value \\
\hline \multirow[t]{10}{*}{ Heavy Chain } & 103 & 112 & YVTYRRYWFD & 10 & -0.925 & 0.000 \\
\hline & 103 & 113 & YVTYRRYWFDY & 10 & -0.595 & 0.000 \\
\hline & 104 & 119 & VTYRRYWFDYWGQGTL & 2 & -0.761 & 0.001 \\
\hline & 104 & 119 & VTYRRYWFDYWGQGTL & 10 & -0.806 & 0.000 \\
\hline & 106 & 119 & YRRYWFDYWGQGTL & 2 & -0.589 & 0.002 \\
\hline & 106 & 119 & YRRYWFDYWGQGTL & 10 & -0.653 & 0.000 \\
\hline & 107 & 119 & RRYWFDYWGQGTL & 10 & -0.592 & 0.000 \\
\hline & 363 & 387 & EEMTKNQVSLTCLVKGFYPSDIAVE & 10 & -0.627 & 0.008 \\
\hline & 387 & 405 & EWESNGQPENNYKTTPPVL & 2 & -0.692 & 0.000 \\
\hline & 388 & 405 & WESNGQPENNYKTTPPVL & 2 & -0.620 & 0.001 \\
\hline \multirow[t]{9}{*}{ Light Chain } & 150 & 173 & KVDNALQSGNSQESVTEQDSKDST & 2 & -1.069 & 0.001 \\
\hline & 150 & 173 & KVDNALQSGNSQESVTEQDSKDST & 10 & -1.101 & 0.000 \\
\hline & 156 & 173 & QSGNSQESVTEQDSKDST & 2 & -0.560 & 0.007 \\
\hline & 163 & 179 & SVTEQDSKDSTYSLSST & 2 & -0.576 & 0.003 \\
\hline & 163 & 179 & SVTEQDSKDSTYSLSST & 10 & -0.580 & 0.003 \\
\hline & 163 & 180 & SVTEQDSKDSTYSLSSTL & 2 & -0.584 & 0.000 \\
\hline & 163 & 180 & SVTEQDSKDSTYSLSSTL & 10 & -0.637 & 0.000 \\
\hline & 168 & 193 & DSKDSTYSLSSTLTLSKADYEKHKVY & 10 & -0.652 & 0.005 \\
\hline & 168 & 195 & DSKDSTYSLSSTLTLSKADYEKHKVYAC & 10 & -0.782 & 0.000 \\
\hline
\end{tabular}

Criteria for significant differences of $\Delta \mathrm{HX}$ :

Wildtype mAb at $\mathrm{pH} 7.0$

Pooled standard deviation $\mathrm{sp}=0.183$, standard error of the mean $\mathrm{SEM}=0.15$, Confidence criteria for $\triangle \mathrm{HX}$ values were calculated by following equation: $|\Delta H X|>k \times S E M$ using $\mathrm{k}=-3.75$ according to Student's t-distribution (One-tailed, $\alpha=0.01, \mathrm{df}=4$ )

Wildtype $\mathrm{mAb}$ at $\mathrm{pH} 5.0$

Pooled standard deviation $\mathrm{sp}=0.208$, standard error of the mean SEM $=0.17$, Confidence criteria for $\triangle \mathrm{HX}$ values were calculated by following equation: $|\Delta H X|>k \times S E M$ using $\mathrm{k}=-3.75$ according to Student's t-distribution (One-tailed, $\alpha=0.01, \mathrm{df}=4$ )

$\mathrm{G}_{27} \mathrm{R}$ variant at $\mathrm{pH} 7.0$

Pooled standard deviation $\mathrm{sp}=0.107$, standard error of the mean SEM $=0.09$, Confidence criteria for $\triangle \mathrm{HX}$ values were calculated by following equation: $|\Delta H X|>k \times S E M$ using $\mathrm{k}=-3.75$ according to Student's t-distribution (One-tailed, $\alpha=0.01, \mathrm{df}=4$ ) 
